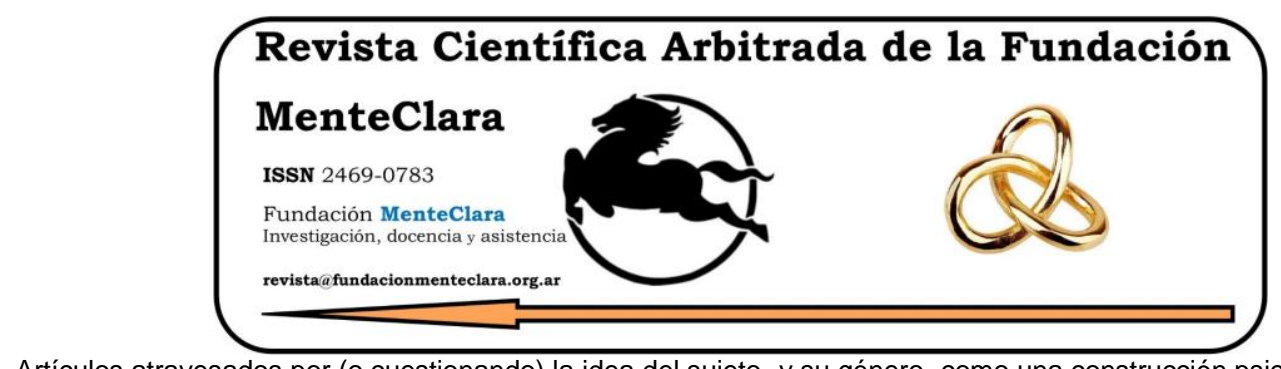

Artículos atravesados por (o cuestionando) la idea del sujeto -y su género- como una construcción psicobiológica de la cultura. Articles driven by (or questioning) the idea of the subject -and their gender- as a cultural psychobiological construction.

Vol. 5 (2020), enero-diciembre

ISSN 2469-0783

https://datahub.io/dataset/2020-5-e201

\title{
UNA REVISION GENERAL SOBRE LA CREATIVIDAD
}

\section{A GENERAL REVIEW ON CREATIVITY}

Patricio Ricci patricio.ricci@uflouniversidad.edu.ar

Facultad de Psicología y Ciencias Sociales, Universidad de Flores, Argentina

Cómo citar este artículo / Citation: Ricci, Patricio. (2020). Una revisión general sobre la creatividad. Revista Científica Arbitrada de la Fundación MenteClara, Vol.5 (201). DOI: https://doi.org/10.32351/rca.v5.201

Copyright: (C) 2020 RCAFMC. Este artículo de acceso abierto es distribuido bajo los términos de la licencia Creative Commons Attribution 4.0 International License (CC BY 4.0). Recibido: 29/11/2020. Aceptado: 08/12/2020 Publicación online: 10/12/2020

Conflicto de intereses: Ninguno que declarar.

\section{Resumen}

En el presente trabajo se intenta dar un panorama general del concepto de creatividad, más allá del conocimiento común del tema y las confusiones o generalizaciones que se suelen dar en su entendimiento. Para ello se recurrió a hacer una revisión y comparación de diferentes investigaciones de relevancia para este trabajo. Se concluyó que la creatividad es un factor de gran importancia para el desarrollo del ser humano a nivel personal y social.

\begin{abstract}
In this paper we try to give a general overview of the concept of creativity, beyond the common knowledge of the subject and the confusions or generalizations that usually occur in its understanding. To do this, a review and comparison of different relevant investigations for this work was used. It was concluded that creativity is a factor of great importance for the development of the human being on a personal and social level.
\end{abstract}

Palabras Claves: Creatividad; Inteligencia

Keywords: Creativity; intelligence 


\section{Introducción}

¿Qué es la creatividad?, ¿Las personas inteligentes son más creativas?, ¿Qué pasa en nuestro cerebro en el acto creativo?, ¿Cómo se puede medir?

Considero a la creatividad clave para poder cambiar aspectos inertes y negativos para las propias personas y para la sociedad a nivel estructural, sin embargo, para el conocimiento común, parece ser algo más bien parecido a un adorno de las personas. Por eso decidí intentar echar luz para esclarecer dicho concepto. Teniendo en cuenta la importancia de la creatividad para diferentes aspectos en el desarrollo humano, en el presente trabajo se intenta dar una aproximación general más fundamentada de lo que se entiende por creatividad en el saber vulgar. Eh recurrido a diferentes investigadores para poder responder a determinadas preguntas básicas. Digo básicas, por que surgen a priori, antes de empezar el estudio. Al abocarme a ellas, surgen muchas otras, más complejas, pretendiendo otro grado de especificidad el cual no es el objetivo en este trabajo.

Con el propósito de hacer una revisión sobre algunos tópicos relacionados a la creatividad y aportar a una idea más fundada sobre este complejo constructo, se dispuso cinco items a desarrollar y en base a eso se procedió a buscar información, mayormente de artículos científicos. Partiendo principalmente de la pertinencia del tema; se verificó su relevancia usando la matriz de información para análisis de revistas "Miar" (http://miar.ub.edu/) y la clasificación según "Circ" (https://www.clasificacioncirc.es/). Luego de la clasificación de gran variedad de artículos, se descartaron los irrelevantes y los de baja fiabilidad, quedando principalmente dos artículos centrales por cada ítem -diez en total-, aunando los conceptos más relevantes para este 
trabajo, sumando aportes de otras fuentes, para lograr más concretamente lo propuesto.

\section{¿Qué es la creatividad?}

Es dificil llegar a una conclusión concreta sobre la Creatividad, debido a que puede abordarse de diferentes ámbitos y para diferentes fines. Además de no ser una capacidad específica, sino más bien, un cumulo de disposiciones. Lara Posada y Correa (2018) sostienen, que, desde una perspectiva coloquial, la creatividad es considerada como la búsqueda de nuevas soluciones a los problemas, logrando algo novedoso a partir de ideas previas. Según Cabrera Cuevas (2018) la creatividad puede tener múltiples significados y evidencia que hace algunas décadas ya se recopilaron más de cuatrocientos significados.

A pesar de todas las formulaciones que ha recibido a lo largo de la historia, la noción de creatividad mayormente hace referencia a la capacidad que poseen las personas para producir ideas originales y, a partir de estas, originar materiales nuevos, tomando siempre como referente el contexto social en el que tienen ocasión esas innovaciones (Muñoz 2011). Para Chiecher et al. (2018) la Creatividad, es; poder formular y resolver problemas de manera divergente, original $y$ alternativa, integrando conocimientos existentes con nuevas perspectivas. Así como para Krumm y Bustos (2014) la creatividad se encuentra entre las más complejas conductas humanas; dice estar influida por una amplia serie de experiencias evolutivas, sociales y educativas, y que se manifiesta de diferentes maneras en una diversidad de campos. Con respecto a esto último, existe consenso entre los investigadores en que la creatividad depende de variados componentes. 
Desde sus diferentes consideraciones, existe un tejido donde la diversidad de ámbitos de aportación profesional, disciplinar, artístico, científico, entre otros, apuestan por una creatividad dirigida hacia el bien común de la humanidad y del planeta (Cabrera Cuevas, 2018).

\section{Relación de la inteligencia con la creatividad}

Normalmente se relaciona la creatividad con diferentes variables como puede ser inteligencia, personalidad y género, entre otras. Aquí vamos a revisar su asociación con la inteligencia, siendo uno de los tópicos más desarrollados del campo. Luego de varios años de estudio, en la actualidad se sigue preguntando sobre las vinculaciones entre estos complejos fenómenos (Elisondo et al. 2010).

La relación entre la creatividad y la inteligencia se ha encarado de diferentes modos, poniendo mucho énfasis en los estudios desde las cinco perspectivas de Sternberg y O`Hara: la creatividad como parte de la inteligencia, la inteligencia como parte de la creatividad, la creatividad y la inteligencia como constructos superpuestos, la inteligencia y la creatividad como lo mismo, y, por último, la creatividad y la inteligencia no se asocian (Sternberg y O`hará, 1999).

Luego de los estudios realizados por Krumm (2014), se comprueba que las asociaciones entre los constructos fueron bajas, aclarando que si bien el Coeficiente Intelectual es necesario para el desarrollo creativo es evidente que la inteligencia por sí sola no puede explicar gran parte de las variaciones de la creatividad.

Un mínimo de inteligencia es necesario para la creatividad, no se observan muestras de puntajes elevados de creatividad y bajos en inteligencia (Elisondo et al., 2010). A partir de cumplir con ese mínimo 
de inteligencia se abre una amplia gama posible entre mayor y menor grado de creatividad. (Corbalán et al., 2003).

Podemos concluir diciendo que hay relaciones variables entres estos dos constructos. Aunque, la dirección de estas relaciones, las influencias mutuas y el comportamiento en diferentes situaciones de la vida sigue siendo poco claro para el campo de investigación de los procesos creativos en particular y de los procesos psicológicos en general (Elisondo y Donolo, 2010).

\section{Relato y aporte desde las neurociencias}

Es necesario conocer más claramente la actividad neuronal latente en los procesos creativos, para poder identificar el contenido biológico y la influencia ambiental sobre esta capacidad cognitiva (Diaz Abrahan y Justel, 2019). Antiguamente se relacionaba directamente al hemisferio derecho con lo creativo. Diversas investigaciones dan cuenta que esta capacidad es parte de un proceso conjunto e integrado entre varios circuitos cerebrales de ambos hemisferios y no solo de uno. Estamos en condiciones de afirmar, entonces que es un proceso que se da de manera global en el cerebro (Muñoz, 2011).

Los principales hallazgos de las neurociencias resaltan la importancia de las regiones prefrontales caudales y rostrales, así como del lóbulo parietal inferior y lóbulo temporal posterior; áreas centrales de los aspectos cognitivos involucrados en la creatividad independientemente del tipo de actividad que se utilice (Díaz Abrahan y Justel, 2019).

Además de describir el funcionamiento cerebral en el proceso creativo, las neurociencias aportan también nuevos conocimientos sobre los bloqueos creativos, identificando posibles causas en la depresión o en la ansiedad. Se vincula las disfunciones del lóbulo frontal con el bloqueo 
creativo o bien a la falta de ideas originales. La depresión es un trastorno que se asocia de manera precisa, a un funcionamiento anómalo de esta región cerebral y, asiduamente, se manifiesta en gran ausencia de motivación y flexibilidad cognitiva. Por cierto, cuando la depresión es tratada clínicamente, se verifica que las funciones del lóbulo frontal se normalizan (Muñoz, 2011).

\section{Una forma de medición}

La investigación sobre creatividad ha crecido de forma muy importante, con nuevos desarrollos teóricos y diversas metodologías (Corbalán, 2010). Para poder tomar una muestra de dichos métodos, y dar una idea de la forma y tamaño de las investigaciones, me interesó centrarme en el Test CREA (Corbalán, Martínez, Donolo, Alonso, Tejerina y Limiñana, 2003). Siendo aún reciente, en el año 2010, ya llevaba más de 3.000 manuales y más de 250.000 ejemplares vendidos, lo que nos permite suponer que aproximadamente unas 230.000 personas han sido evaluadas con él, en solo siete años (Corbalán y Limiñana-Gras, 2010). Si en un solo test, podemos constar de tantas muestras, damos cuenta del interés de los investigadores por el estudio de la Creatividad.

¿Cómo se realiza el test?:

El CREA ofrece una medida indirecta de la creatividad que utiliza como indicador a la capacidad de las personas de formular preguntas ante tres estímulos visuales «A, B y C». En este sentido, el TEST CREA permite la evaluación de la dimensión verbal de la creatividad. En la lámina A puede observase un teléfono antiguo; las láminas B y C representan situaciones extrañas o absurdas. Se trata de una prueba de ejecución máxima, la consigna general es: "Se te va a presentar una ilustración. Tu tarea 
consiste en escribir brevemente todas las preguntas que puedas hacer sobre lo que representa la lámina. Trata de hacer el mayor número posible de preguntas". Las personas disponen de cuatro minutos para realizar el mayor número posible de preguntas para cada lámina. El CREA es de aplicación individual para niños de 6 a 9 años. En niños mayores de 9 años, adolescentes y adultos la aplicación puede ser individual o colectiva. Se valora la cantidad de preguntas formuladas por las personas, se asignan puntajes adicionales para interrogantes que incluyen diferentes esquemas y contenidos. En el manual se presentan baremos para niños, adolescentes y adultos de población española y argentina, también se incluyen pautas que permiten clasificar a las producciones según tres criterios: creatividad alta, media o baja (Elisondo y Donolo, 2018).

El CREA no abarca ni pretende abarcar todo el campo del estudio de la creatividad, pero ciertamente, ofrece alternativas eficientes para conocer determinados aspectos de mucha importancia para la misma y se muestra como un predictor de la capacidad creativa de las personas y en ocasiones además de poner en manifiesto las conductas creativas habituales, da a conocer potencialidades inhibidas, ocultas o desconocidas en sus poseedores (Corbalan y Limiñana-Gras, 2010).

\section{La creatividad en su campo natural}

Los ambientes de los estudios científicos, propiamente controlados, como también así las diferentes formas de medición realizadas por los investigadores -como podría ser el Test, nombrado anteriormente- que contemplan evaluaciones de la variable creatividad, imponen dificultades y desafios, ya que el mismo ambiente e intención del investigador, puede poner en riesgo y afectar al proceso de creación y por lo tanto la validez 
de las observaciones (Abrahan y Justel, 2019). Por ello eh decidido aportar aquí algunos datos sobre la investigación de la variable, teniendo en cuenta uno de los ámbitos naturales de su desarrollo, la composición musical.

Las áreas artísticas como pueden ser la música, la danza o el teatro son de vital importancia para el estudio de la creatividad. Por este motivo muchas investigaciones se basan en dichas habilidades, tomándolas como factores ambientales que modulan la capacidad creativa (Abrahan y Justel, 2019). En estas áreas, los participantes no son tratados como objetos experimentales para el uso de la investigación, sino más bien, los participantes desarrollan su práctica libremente, sin ser condiciona más que por sus propias limitaciones, y una vez terminada es relatada a el investigador (Lara y De Castro Correa, 2018). La creatividad surge en ellos como una tensión entre la espontaneidad, el contexto cultural y sus limitaciones.

Al componer una obra musical, es necesario un proceso consciente y racional que le hace dar cuenta al compositor sobre los propios recursos con los que cuenta, como así también, los que no posee (Lara y De Castro Correa, 2018). A partir de ese proceso racional o consciente, puede entonces empezar a organizar los datos sensoriales que comienzan a surgir desde una primera etapa intuitiva. (Lara y De Castro Correa, 2017). A partir de la elaboración y organización de formas sonoras se logra exteriorizar las ideas inmersas en las experiencias emocionales, así, los compositores logran mayor claridad y dominio en el conocimiento de sus deseos. Siendo la voluntad el acto de asumir la responsabilidad a partir de la cual se ponen en práctica las elecciones y clarificar con cuales deseos se identifican (Lara y De Castro Correa, 2018). El análisis de la intencionalidad, además de implicar y partir de la forma de vivenciar los deseos, también debe implicar y partir de la forma en que el ser humano 
recurre a su voluntad para relacionarse o conectarse con sus deseos (De Castro \& García, 2011).

La neurosis halla su núcleo al encontrar que el hombre se ve responsable de tomar decisiones (May, 2000). En contraste a esto, a partir de las observaciones y de las experiencias analizadas de los compositores, visibilizan como, mediante sus intenciones, decisiones actuales y formas de vivenciar sus deseos, participan y contribuyen activamente en la construcción de sus deseos. La composición musical les aporta sensación de sentido coherente en diferentes dimensiones de sus vidas, brindándoles bienestar psicológico que infiere en su salud mental (Lara y De Castro Correa, 2018).

Por otra parte, en referencia a la neurosis y la creación artística, Sigmund Freud, expresó:

"En la creación artística misma, se discernió que el ámbito de la fantasía era como una "reserva natural" instituida a raiz del paso, sentido dolorosamente, del principio de placer al de realidad, a fin de proveer un sustituto a la satisfacción pulsional que debió resignarse en la vida real y efectiva. El artista, como el neurótico, se habia retirado de la insatisfactoria realidad efectiva a ese ámbito de la fantasía, pero, a diferencia de aquel, se ingeniaba para hallar el camino de regreso $y$ volver a hacer pie sólidamente en la realidad fáctica" (Freud, 1925).

\section{Conclusión}

Eh hecho solo un breve repaso por algunos tópicos relacionados a la creatividad, sin embargo, ya desde este trabajo, se puede concluir en la importancia que tiene el estudio de dicha variable, debido a su influencia positiva en el desarrollo humano tanto a nivel personal, como a nivel social. Si bien en los últimos años se ha incrementado el estudio de la 
misma, aún quedan muchos espacios por ocupar, que pueden llegar a dar luz a cuantiosos avances en el desarrollo integral de las personas y sus comunidades.

“La existencia auténtica, por su parte, denota el modo de ser en el que el hombre comprende que él es posibilidad, que puede apropiarse y responsabilizarse de su existencia; en la autenticidad el hombre se resuelve, elige adueñarse genuinamente de las posibilidades que se le abren" (Heidegger, 1997). 


\section{Referencias}

Cabrera Cuevas, Jessica (2018). Epistemología de la creatividad desde un enfoque de complejidad. Educación y Humanismo. 20. 113-126.

https://doi.org/10.17081/eduhum.20.35.3127

Chiecher, A., Elisondo, R., Paoloni, P., \& Donolo, D. (2018). Creatividad, género y rendimiento académico en ingresantes de ingeniería. Revista iberoamericana de educación superior, 9(24),138 151.

https://doi.org/10.22201/iisue.20072872e.2018.24.269

Corbalán, J. \& Limiñana-Gras, R. (2010). El genio en una botella. El test CREA, las preguntas y la creatividad. Introducción al monográfico "El test CREA, inteligencia creativa". Anales de Psicología. 26. 197-205.

Corbalán Berná, J., Martínez Zaragoza, F., Donolo, D., Alonso Monreal, C., Tejerina Arreal, M. \& Limiñana Gras, M.R. (2003). CREA. Inteligencia Creativa. Una medida Cognitiva de la Creatividad. TEA Ediciones. Madrid.

De Castro, A. \& García, G. (2011). Psicología Clinica: Fundamentos Existenciales. Barranquilla: Ediciones Uninorte.

Diaz Abrahan, V. \& Justel, N. (2019). Creatividad. Una revisión descriptiva sobre nuestra capacidad de invención e innovación. CES Psicología. 12. 35-49. 10.21615/cesp.12.3.3.

Elisondo, Romina \& Donolo, Danilo. (2010). ¿Creatividad o inteligencia? That is not the question. Anales de Psicología. 26.

Elisondo, Romina \& Donolo, Danilo. (2018). Preguntones y creativos: discusiones y evidencias. Psicodebate. 18. 35. https://doi.org/10.18682/pd.v18i1.715

Freud, S. (1925/1926). Presentación autobiográfica, Obras Completas XX. Bs. As.: Amorrortu.

Heidegger, M. (1997). Ser y tiempo. Buenos Aires: Fondo de Cultura Económica.

Krumm, G., Arán Filippetti, V., \& Bustos, D. (2014). Inteligencia y creatividad: correlatos entre los constructos a través de dos estudios empíricos. Universitas Psychologica, 13(4), 1531-2143. https://doi.org/10.11144/Javeriana.UPSY13-4.iccc

Lara-Posada, E \& Correa, A. (2017). Libertad vs. Límites en la creación de una obra musical. Psicología desde el caribe. 34. 172-183.

https://doi.org/10.14482/psdc.33.2.72786. 
Lara Posada, E. \& Correa, A. (2018). Intencionalidad en la creación de una obra musical. CES Psicologia. 11(1), 102-117. https://doi.org/10.21615/cesp.11.1.8

May, R. (2000). Amor y voluntad, Las fuerzas que dan sentido a nuestra vida. Gedisa.

Muñoz, F. (2011). Construcciones de la neurociencia al entendimiento de la creatividad humana. Arte, Individuo y Sociedad. 23. https://doi.org/fs95v9

Sterberg, R., \& O`Hara, L. (1999). Creativity and intelligence. En R. J. Sternberg (Ed.), Handbook of creativity (pp. 251-272). Cambridge University Press. 